\title{
Impact of intensive nutritional education with carbohydrate counting on diabetes control in type 2 diabetic patients
}

This article was published in the following Dove Press journal:

Patient Preference and Adherence

23 December 2010

Number of times this article has been viewed

\section{Christopher Zipp \\ Jessica Terrone Roehr \\ Lucia Beck Weiss \\ Frank Filipetto}

Department of Family Medicine, School of Osteopathic Medicine, University of Medicine and Dentistry of New Jersey, Stratford, NJ, USA
Correspondence: Christopher Zipp Department of Family Medicine, School of Osteopathic Medicine, University of Medicine and Dentistry of New Jersey, 42 East Laurel Road, Stratford,

NJ 08084, USA

$\mathrm{Tel}+\mathrm{I} 8565666359$

Fax +I 8565666360

Email zippch@umdnj.edu

\begin{abstract}
This pilot study assessed the impact of an intensive carbohydrate counting educational intervention on diabetes control in type 2 diabetic patients. An experimental, prospective study design was used to assess the effect of nutritional education on diabetes control. The impact and efficacy of the education were measured over a 1-year period through changes in diabetes clinical markers, including hemoglobin $A_{1 c}$, lipid profiles, glucose levels, patients' energy levels, and sense of well-being. Six patients were initially enrolled in the pilot study, with only three patients completing the intervention phase and the 3-month follow-up. Two patients were followed-up at the 1-year mark for their diabetes, although neither continued participation in the study beyond the 3-month mark. Marginal improvements in clinical markers at 3 months were found. However, due to the small sample size, changes in the clinical profiles may have occurred because of variables unrelated to the nutritional intervention. Further research is indicated for the control of these variables.
\end{abstract}

Keywords: type 2 diabetes, nutritional education, carbohydrate counting, diabetes control

\section{Introduction}

Diabetes mellitus continues to be an ever-growing chronic condition facing the American population. The latest data published from the Centers for Disease Control and Prevention (CDC) show that the prevalence of both diagnosed and undiagnosed diabetes is now approaching 24 million people. ${ }^{1}$ Diabetes is the seventh leading cause of death in the United States, and the risk of death for patients with diabetes has been reported to be twice that of similar people in the same age group without diabetes. ${ }^{1}$ As a result of these risks and increasing prevalence of diabetes, additional research is needed that can identify effective strategies to control this chronic condition and improve patient outcomes.

Many multidisciplinary strategies, including the use of general diabetes education interventions, along with diabetes self-management goal setting, self-monitoring of blood glucose, and nutritional education, have been well-documented in the literature demonstrating improvements in glycemic control and other diabetes clinical outcomes. ${ }^{2}$ However, minimal practice-based research exists that directly shows the benefit of intense nutritional counseling with a focus on carbohydrate counting to improve diabetes outcomes. ${ }^{2}$

Developing and adhering to a specific nutritional plan is one of the key factors for optimal diabetes control. However, it can be one of the most challenging aspects of management for both the patient and the clinician. ${ }^{3}$ Patients often have difficulty adhering to a specific diet based on individual lifestyle schedules, habits, 
and routines, as well as personal and cultural food choices and preferences. ${ }^{3}$ As a result, carbohydrate counting may be a solution for patients, as it offers a meal-planning approach and not a specific set of guidelines for one type of diet. ${ }^{3,4}$

It has been shown that the regulation of carbohydrates by a variety of methods, one being carbohydrate counting, is a key strategy for optimal glycemic control. ${ }^{5}$ Carbohydrate counting requires intense patient education by health care providers, specifically registered dietitians, and certified diabetes educators. It can be broken down into two types: basic and advanced. ${ }^{2,4}$ Basic carbohydrate counting is based on learning general relationships between food, carbohydrate consistency, portion sizes, blood glucose levels, and physical activity. ${ }^{4,6}$ Advanced carbohydrate counting for patients requiring insulin involves using insulin to carbohydrate ratios tailored to patients' target blood glucose levels. ${ }^{4}$ It involves adjusting the amount of insulin based upon the amount of carbohydrates ingested in grams, using the standard conversion of one carbohydrate serving being equivalent to $15 \mathrm{~g}$ of carbohydrate. ${ }^{4}$

As a result of these factors, a small pilot study was conducted to determine the impact of an intensive carbohydrate counting educational intervention on type 2 diabetes control.

\section{Patients and methods}

An experimental, Institutional Review Board-approved, prospective study design was used to assess the effect of a carbohydrate counting nutritional education on diabetes control. The impact and effectiveness of the education was measured primarily through changes in diabetes clinical markers and patients' overall sense of health and well-being, with planned monitoring for a 1-year period. In order to be enrolled in the study, participants should have completed a general nutrition group education training component, which would be followed by a 3-month intensive nutrition education program for each participant. Clinical markers were assessed prior to the nutritional intervention and at 3, 6, and 12 months following the intervention.

This pilot study sought to recruit 10 subjects from an established primary care patient population. Eligibility for inclusion consisted of having a diagnosis of diabetes mellitus type 2 and being an English-speaking patient aged over 18 years. Participation in this research study was voluntary.

Eligible patients who were either insulin- or noninsulindependent type 2 diabetics were enrolled in the study through the efforts of the lead researcher. Prior to being enrolled in this pilot study, each study subject had participated in a nutrition group education program conducted by a registered dietitian and certified diabetes educator. As part of that program, subjects received instruction about general diabetes nutrition and were trained in the proper use of a glucometer for self-monitoring of blood glucose levels. Upon enrollment in the study, participants were to receive additional basic carbohydrate counting education through a group session or one-on-one instruction.

Following this instruction, a 3-month intensive intervention was initiated. During this 3-month period, participants were instructed to keep a daily $\log$ (Appendix A) of fasting, preprandial, and postprandial blood glucose along with the number and types of carbohydrates they consumed for breakfast, lunch, dinner, and two snacks. Participants self-reported their energy levels after each meal based on a provided scale. Regular teaching intercession and follow-up telephone appointments were held with each subject to reinforce concepts learned and to assess compliance through phone calls, surveys, and monthly office visits.

During the month following the educational intervention, weekly telephone sessions were conducted with each participant by one of the study researchers. During these telephone sessions, a brief review of carbohydrate counting was provided to the participant and comprehension was measured by whether or not the participant was able to teach back the concepts. The participant's log was reviewed, and the subject discussed their meal-planning strategies with the study researcher.

During the second and third months, biweekly phone calls were made to each subject for additional follow-up and reinforcement. In addition, during this 3-month intervention, monthly office visits were planned for the subjects, where face-to-face follow-up would be made, daily logs collected, and survey questionnaires distributed to assess overall well-being (Appendix B).

Throughout the intervention period, a dedicated phone number was available to study participants for asking questions related to the intervention. A password-protected voice mail system would allow participants to leave messages for the study researchers. Study researchers would contact participants and address questions within 48 hours.

Clinical markers were also assessed throughout this study. Hemoglobin $\mathrm{A}_{1 \mathrm{c}}$, fasting, preprandial and postprandial glucose, lipid profiles, and overall sense of well-being were to be assessed before the educational sessions as well as at 3, 6, and 12 months following the intensive intervention. 


\section{Results}

Nine patients were recruited by the primary investigator (PI) during office visits to participate in the initial educational event. Despite reminder calls, only five participants attended this initial event. Additional enrollment was sought by the PI and research coinvestigator. Participation in the study was offered to additional patients who met the study parameters during office visits. One additional subject was enrolled in this manner, who then received the initial educational training on a one-on-one basis. As a result, six patients were actively enrolled in the study.

Four of these six participants completed the entire 3-month intensive intervention. Depsite multiple attempts to perform monthly face-to-face evaluations, these four participants followed up at varying intervals. Two out of the initial six participants voluntarily withdrew from the study, each stating that it was too difficult to follow the regimen.

Of the four patients who participated in the 3-month intensive educational intervention, three showed an improvement in their hemoglobin $\mathrm{A}_{1 \mathrm{c}}$ with a $>1 \%$ decrease at the 12-month follow-up (Table 1). A dramatic improvement in the lipid profile was seen in one study participant at 12 months; however, little to no improvement was seen in other participants (Table 1). Preprandial glucose levels initially improved in patient 1 , varied for patient 2 , and improved for patients 3 and 4 (Table 1). No patient reported postprandial glucose levels despite attempts by researchers to reinforce this need.

The researchers developed a simple Likert-type scale ranging from 0 to 8 assessing self-reported energy levels and sense of well-being during the 3-month intervention period. This was not a validated instrument, as participants self-rated their own perceived energy levels and sense of well-being, and data were not used to compare participants to one another. Patients were asked to record energy levels on the Likert-type scale, where energy levels recorded on a daily basis ranged from 4 to 6 across the intervention period for patient 1 , indicating average to moderate energy levels. Levels ranged from 6 to 8 for patient 2, with readings consistently at level 8 at the end of the 3-month intervention, indicating moderate energy to feeling energized most days at the end of the intervention. Patient 3 recorded energy levels of 6 , which is moderate energy, for the majority of the intervention phase.

The survey administered to the six participants at baseline to establish health and well-being yielded the following results (Table 2). The first question assessed if patients felt healthy. One disagreed, one was neutral, three agreed, and

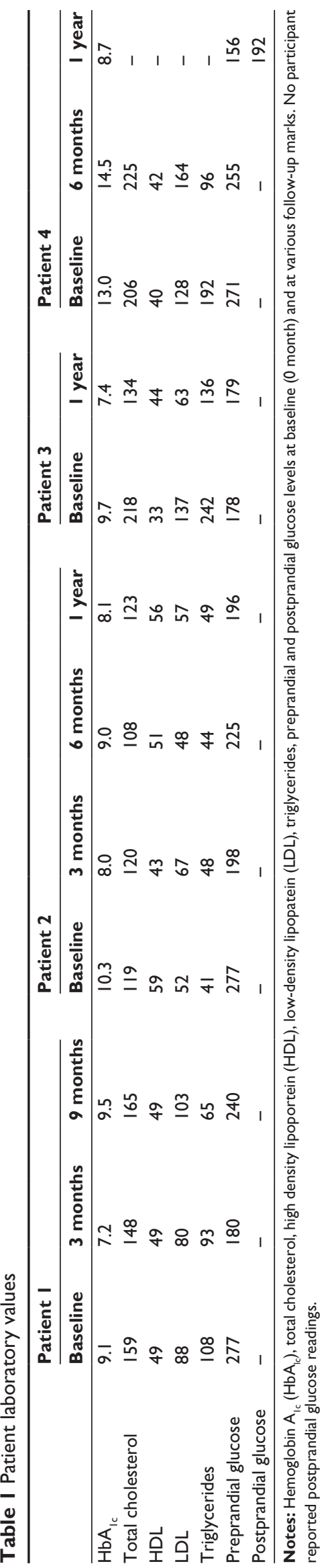




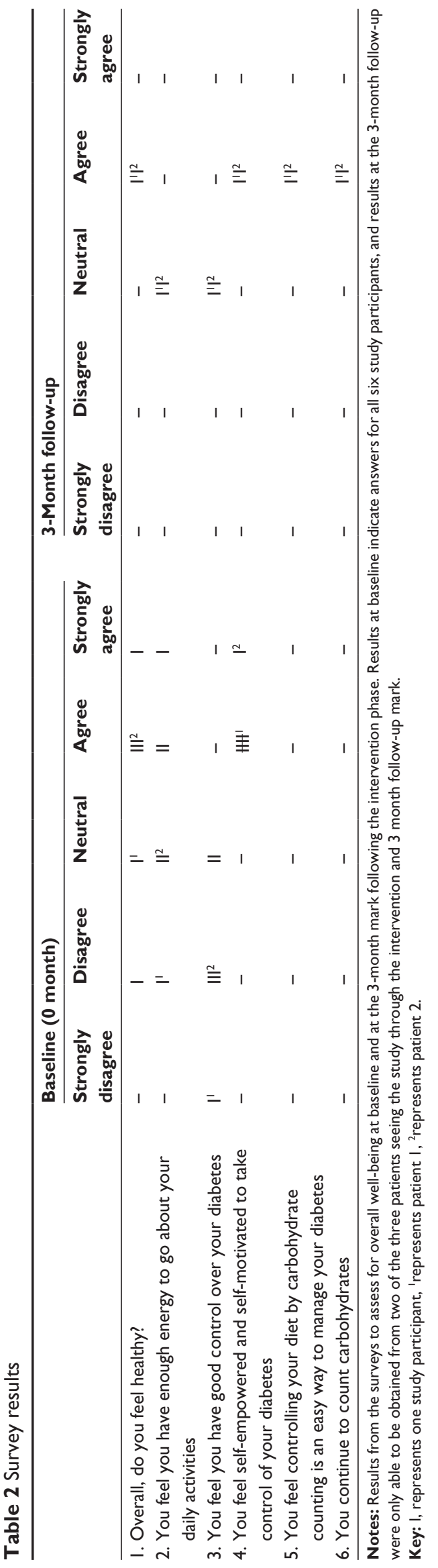

one strongly agreed. The second question assessed if patients had enough energy to go about their daily activities. One disagreed, two were neutral, two agreed, and one strongly agreed. The third question assessed if patients felt they had good control over their diabetes. One strongly disagreed, three disagreed, and two were neutral. All of the participants agreed they felt self-empowered and self-motivated to take control of their diabetes.

Only two of the four participants completed the 3-month follow-up survey (Table 2). These two patients agreed to feeling healthier at 3 months compared to baseline. At the 3-month follow-up, both patients had an improved perception of diabetes control. Improved control of diabetes was also reflected in the interval improvement of each patient's hemoglobin $\mathrm{A}_{\mathrm{lc}}$. Both patients indicated that they 'agree' to feeling self-empowered and self-motivated to take control of their diabetes at the 3-month follow-up mark. Both patients agreed that controlling their diet by carbohydrate counting is an easy way to manage diabetes and they would continue to count carbohydrates.

The dedicated phone number that was set up for participants to leave messages for the study researchers did not receive any use throughout the study, despite subjects being aware and reminded that it was available if needed. In addition, surveys and monthly office visits during the intervention phase were not administered to all patients as had been delineated in the study protocol due to the patients' preferences.

\section{Discussion}

Results indicate only marginal improvement of diabetic control for patients enrolled in this small study. Researchers feel this was mostly due to the lack of follow-up by participants. This occurred despite patients indicating they felt self-empowered and self-motivated to take control of their diabetes. Although some improvements in hemoglobin $\mathrm{A}_{\mathrm{lc}}$ and lipid profiles were observed, conclusions cannot be made based on the small number of participants and inconsistent participation of enrollees. In addition, it is difficult to attribute both favorable and unfavorable outcomes to carbohydrate counting alone as other variables may have contributed to the results. Studies for the control of these variables should be carried out.

Patient 4 was able to achieve a significant improvement in hemoglobin $\mathrm{A}_{\mathrm{lc}}$ and lipid profile at the 12-month follow-up due to the support provided by physicians, nurses, and dietitians during a 2-month hospitalization that occurred in months 10 and 11 . 
The cohort of patients in this study did not appear to be interested in follow-up, despite reminder phone calls from the researches and even from the patients' primary care provider. Stated reasons for poor compliance included patients' inability to balance the rigorous nature of the study within their daily life and disinterest. These outcomes, as other studies have indicated, show that making lifestyle changes including nutritional choices is a difficult task for many patients. A program geared for patients with chronic disease must take into consideration the individual patient, their specific life circumstances, and barriers to effective outcomes.

Future work should continue as diabetes continues to be a growing chronic disease in the United States. Future studies beyond this small pilot study may show that intensive education does make a difference in patient outcomes. Despite poor patient compliance and long-term follow-up, feedback by patients with biweekly phone sessions during the intervention phase was strongly positive. Multiple patients stated that they liked the individual follow-up, teaching, and support. Perhaps this component of individual follow-up and reinforcement could be incorporated into further studies with chronic disease populations.

\section{Conclusion}

The use of intensive nutritional education with carbohydrate counting on diabetes control in type 2 diabetic patients may yield improvement in clinical outcomes. However, the study as conducted shows that barriers continue to exist regarding diabetes outcomes despite attempts at educational interventions. Future work in this area, with changes to this pilot study design, may produce more positive outcomes.

\section{Disclosure}

The authors report no conflicts of interest in this work.

\section{References}

1. Centers for Disease Control and Prevention. Available from: http://www. cdc.gov/diabetes/pubs/pdf/ndfs_2007.pdf. Accessed Nov 102010.

2. Pastors JG, Warshaw H, Daly A, Franz M, Kulkarni K. The evidence for the effectiveness of medical nutrition therapy in diabetes management. Diabetes Care. 2002;25(3):608-613.

3. Rabasa-Lhoret R, Garon J, Langelier H, Poisson D, Chiasson JL. Effects of meal carbohydrate content on insulin requirements in type 1 diabetic patients treated intensively with the basal-bolus (ultralente-regular) insulin regimen. Diabetes Care. 1999;22(5):667-673.

4. Kulkarni KD. Carbohydrate counting: a practical meal-planning option for people with diabetes. Clin Diabetes. 2005;23(3):120-122.

5. Gillespie SJ, Kulkarni KD, Daly AE. Using carbohydrate counting in diabetes clinical practice. J Am Diet Assoc. 1998;98(8):897-905.

6. Piziak VK. Medical management of obesity. Promoting healthy dieting. Postgrad Med. 1983;74(5):158-166, 171-173.

7. Makrilakis K, Katsilambros N. Prediction and prevention of type 2 diabetes. Hormones (Athens). 2003;2(1):22-34.

8. Carels RA, Young KM, Coit CB, et al. The failure of therapist assistance and stepped-care to improve weight loss outcomes. Obesity (Silver Spring). 2008;16(6):1460-1462. 


\section{Appendix A}

Sample sheet of one day from subject log book. This was created into a personal book for each subject with one page given per day. The books were collected each month and each subject received three books to track their progress for the three month intensive intervention.

Log Book

Date

Fasting Blood Sugar

\begin{tabular}{|l|l|l|l|l|}
\hline Meal & $\begin{array}{l}\text { Preprandial sugar } \\
\text { (Premeal sugar) }\end{array}$ & Carbohydrates & $\begin{array}{l}\text { Postprandial Sugar } \\
\text { (Postmeal sugar) }\end{array}$ & $\begin{array}{l}\text { Energy } \\
\text { Level }\end{array}$ \\
\hline Breakfast & & & & 02468 \\
Lunch & & & & 02468 \\
Dinner & & & & 02468 \\
Snack \#I & & & & 02468 \\
Snack \#2 & & & 02468 \\
\hline
\end{tabular}

Energy Level Key

$0=$ very fatigued; $2=$ moderately fatigued; $4=$ average; $6=$ moderately energized; $8=$ energized

\section{Appendix B}

Survey planned to be distributed initially at baseline, at monthly office visits, and at 3-, 6-, and 12-month intervals following the intervention to assess overall well-being.

Subject Name

Please circle one of the following choices for each statement.

1) Overall, do you feel healthy?

Strongly Disagree Disagree Neutral Agree Strongly Agree

2) You feel you have enough energy to go about your daily activities.

Strongly Disagree Disagree Neutral Agree Strongly Agree

3) You feel that you have good control of your diabetes.

Strongly Disagree Disagree Neutral Agree Strongly Agree

4) You feel self-empowered and self-motivated to take control of your diabetes.

Strongly Disagree Disagree Neutral Agree Strongly Agree

*Statement added to survey for the monthly office visits during the intensive intervention phase of the study:

5) You feel controlling your diet by carbohydrate counting is an easy way to manage your diabetes.

Strongly Disagree Disagree Neutral Agree Strongly Agree

*Statement added for the 3-, 6-, and 12-month intervals following the intervention:

6) You continue to count carbohydrates.

Strongly Disagree Disagree Neutral Agree Strongly Agree

Patient Preference and Adherence

Dovepress

\section{Publish your work in this journal}

Patient Preference and Adherence is an international, peer-reviewed, open access journal that focusing on the growing importance of patient preference and adherence throughout the therapeutic continuum. Patient satisfaction, acceptability, quality of life, compliance, persistence and their role in developing new therapeutic modalities and compounds to optimize clinical outcomes for existing disease states are major areas of interest. This journal has been accepted for indexing on PubMed Central. The manuscript management system is completely online and includes a very quick and fair peer-review system. Visit http://www.dovepress.com/ testimonials.php to read real quotes from published authors. 